\title{
Critical Study
}

\section{Trudy Govier's Problems in Argument Analysis and Evaluation}

\author{
DEREK ALLEN University of Toronto
}

Govier's concerns in this work are both critical and constructive. Her chief critical concern is to demonstrate that certain current theories of argument are inadequate, while her chief constructive concern is to contribute to the development of a better theory.

The book is written with the lucidity for which Govier is renowned. It displays intimate familiarity with the informal logic literature. And it covers a wide range of topics. On each, Govier has interesting things to say, as readers of this journal would expect.

It is unfortunate, then, that the text is marred by an extraordinary number of typographical errors, some of which seriously distort its meaning. It is also unfortunate that, of the thirteen chapters, there are, after the first, only four each of which seems naturally to follow the one that precedes it. Some judicious reordering, and some additional bridging, would have given the book a tighter knit.

\section{(1) A Summary:}

In her opening chapter Govier argues that formal logic provides an inadequate basis for the assessment of actual arguments. Of course no formal logician would seriously maintain otherwise. Nevertheless, formal logic "seeks to maintain its authority as the ultimate standard against which real arguments should somehow be measured"' (9). ${ }^{1}$ Formally valid arguments function as a kind of paradigm - a paradigm which "blinds" formally trained logicians and philosophers to the obvious fact that "real argumentation is not easily or usefully amenable to formal treatment" (10). Against this paradigm, Govier recommends a revolution.

For those blinded by the paradigm, logic is formal logic and formal logic is a science-the science of argument assessment. But argument assessment, Govier insists, is "more an art than a science" (203). The same is true of argument interpretation. Neither argument interpretation nor argument assessment, then, can be mechanized; that is to say, neither can "be captured by a set of rules which set out a mechanical decision procedure" (221). There is an argument to the contrary, however, which maintains in part that for any intelligent human activity "there is a rule or set of rules describing how that activity is done or should be done" (220). But intelligent human activity, Govier responds, may occur according to a ceteris paribus rule, and a rule of this sort cannot be used mechanically for the simple reason that it is impossible to spell out "all the unusual circumstances that would make the rule inapplicable" (223). The use of a ceteris paribus rule requires judgment. So do the activities of argument interpretation and argument assessment. Neither can be done by applying rules. To be sure, the appraisal of inferences-" $[t]$ he primary task of logic" 
(209) - may involve the application of rules. But to know what rules to apply to a particular argument, we must first classify the argument as one of a type, and this is not a matter of applying rules: it requires a sense of how the argument is supposed to work. Moreover there is reason to question "the feasibility or desirability of formally expressing rules for material inferences" (211). This is evident from the technique of using a logical analogy to refute an argument with the same logical structure, for the technique typically serves to isolate as the structure of an argument something that is not a formal structure, in any standard sense of 'formal', but ' a meaning structure" (213). Accordingly, the judgments that logical analogies enable us to make of material inferences are nonformal judgments. Can we rest content with such judgments or should we construct formal systems to make them more precise? This same question arises when we consider the common claim that informal fallacies must be given a formal analysis if they are to be properly understood. That it would be posble in principle to develop a formal theory of the informal fallacies, Govier does not deny. But the development of such a theory would tend to conceal controversial assumptions in definitions and rules-assumptions we would unavoidably make in explaining why a particular informal fallacy is a fallacy. "[M]uch reasoning would go on outside that theory and precious little within it' (220), for there are controversial questions pertaining to informal fallacies that a formal theory is powerless to answer.

Similarly, there are controversial questions about arguing and argument that lie beyond the bounds of formal logic-such as "How many different types of arguments are there?', 'When and why should we regard an argument as having missing or unstated premises?', and 'Is the truth of premises too strong a condition to demand for soundness of argumentation?', ' (13). These are questions for a nonformal theory of argument.
But a nonformal theory of argument is an impossibility if, as McPeck has argued, any general account of argument would be a formal account. Now admittedly a theory of natural argument would be formal in one sense of the word if it were a general, systematic and well-ordered theory. But this need not trouble informal logicians. For they are committed to the view that a theory of natural argument would not be formal in a different sense-the sense in which formal logic is formal. And McPeck has not shown that this view is mistaken. Indeed he himself has a theory of argument that is in the requisite sense nonformal-a disciplinespecific theory according to which natural arguments are properly assessed just by reference to standards of one or more specific disciplines. But a radical disciplinespecific theory leaves much to be desired: it is difficult to apply, it fails to give "a natural description of the phenomena of argument" (21), and it is implicitly uncritical and conservative.

There are, of course, other theories of argument around. Govier considers three, and finds each wanting. One is deductivism-the view that a good argument must be deductively valid, either in virtue of its logical form (formal deductivism) or in virtue of meaning or form (non-formal deductivism). Literal deductivism has the consequence that "all invalid arguments are equally and totally flawed"' (25). Since most natural arguments are not, as stated, deductively valid, the literal deductivist cannot escape the skeptical conclusion that most natural arguments are bad arguments. But deductivism may be reconstructive rather than literal. Reconstructive deductivists regard as incomplete all natural arguments which as stated are not deductively valid and recommend reconstructing such arguments as deductively valid arguments by adding appropriate 'missing' premises. While this approach is preferable in Govier's view to that of literal deductivism, she still finds it "costly" (see below, Section 2). And it is not clear that the costs are worth paying if, 
as Govier complains, deductivists have given us no good reason to accept their central claim-that a good argument must be deductively valid.

For deductivism there are no degrees of logical support. But for "the spectrum theory", there are: one argument may provide a greater degree of support for its conclusion than another. But what are degrees of support degrees of? And what constitutes a greater or lesser degree? The spectrum theory fails to answer these questions, and Govier therefore dismisses it as a metaphor.

The spectrum theory isn't very common. But the positivist theory is. This is the theory that all arguments are either deductive or inductive. There are different ways of explaining the deductive/inductive distinction, of which the most commonly accepted by philosophers is Copi's. According to Copi, a deductive argument claims that its premises entail its conclusion, while an inductive argument claims that its premises (if true) make its conclusion probable. But, drawn in this way, the deductive/ inductive distinction is difficult to apply to real arguments because it is often difficult to decide whether an argument is 'claiming' a necessary or probabilistic connection between its premises and conclusion. Thus the positivist theory has a classification problem. But this does not count decisively against it, because classification problems are also likely to beset other pluralistic theories of argument. The key issue for the positivist theory, rather, is whether there is only one kind of nondeductive connection between premises and conclusion-to wit, a probabilistic connection. If a probabilistic connection is simply nondeductive, the category has little content. But if it is interpreted more narrowly, as an empirical relation, then the positivist theory will leave no room for "nondeductive arguments in which the conclusion and relation of premises to conclusion is non-empirical" (52). And this is "the real danger of the great divide" (53) in the theory: it leads to "false simplicity" in our classificatory scheme by making it "too easy to ignore the many nondeductive arguments which are not classically inductive" (53).

Govier has in mind here two species of argument in particular: argument by a priori analogy and conductive argument (see below, Sections 3 and 4). Both have been widely ignored by logicians and philosophers, perhaps because neither is fully susceptible of treatment by general rules. An adequate theory of argument would recognize their existence. Since it would obviously recognize deductive and (classically) inductive arguments too, an adequate theory of argument would be more pluralistic than the positivist theory.

Govier's own pluralism underlies certain critical stances she adopts in a chapter on fallacies. There she examines a skeptical view about informal fallacies held by Karl Lambert and William Ulrich. For them, "only formal invalidity constitutes a mistake in reasoning" (179). If we are able to show that an argument is invalid, it will serve no point to add that the argument also exemplifies an informal fallacy, for this will have no bearing on the identification or analysis of the mistake in reasoning involved in it. By way of illustration, Lambert and Ulrich discuss ad hominem. Govier remarks that the discussion "presumes a very narrow theory of argument" (a "formalist and deductivist" theory) and argues that, even within the framework of that theory, the discussion "raises as many questions as it answers" (181). What is more, she claims, ad hominems share a feature that has to do with their content, not with their formal structure. Lambert and Ulrich, however, claim that arguments with this feature may be deductively valid. Govier agrees, but says that this goes to show that "assessing arguments solely [sic] for formal deductive validity will sometimes show us precious little about their merits" (183). It also shows that invalidity is not a necessary condition for fallaciousness. Moreover, from the perspective of a pluralist theory, neither is 
it a sufficient condition.

Lambert and Ulrich assume that it is possible to prove an argument invalid. Gerald Massey has of course argued otherwise, and connected the point to fallacies. He makes the connection apparent in an argument which runs roughly as follows. Fallacies are invalid arguments. To show that an argument is a fallacy, therefore, we must show that it is invalid. But there is no formally adequate way of showing that an argument is invalid. Consequently, we cannot show that an argument is invalid in any theoretically adequate way. Hence there is no adequate theory underlying fallacies. Govier objects that "[f]rom the perspective of a pluralistic theory of argument and a nonformalist concept of theoretical adequacy", this argument is far from conclusive. "The problem is in moving from not having a formally adequate method to not having any theoretically adequate method . . . Anyone who thinks that respectable nonformal theories are possible will not accept this move" (188).

Lambert, Ulrich and Massey at least do not deny that there are fallacies. But, impressed by what he regards as inadequate textbook treatments of the subject, Maurice Finocchiaro has concluded that "there are probably no real fallacies in practice; that fallacies exist only in the minds of pretentious logicians" (191). He believes that those who claim to find fallacies misinterpret natural reasoning by "exaggerating the strength of the connection claimed between various assertions, or . . . [by] creating one where none is claimed" (196). This criticism, Govier charges, "rests on a controversial and undefended theory of argument" (196)-viz., a version of the spectrum theory. As for the claim that textbook treatments of fallacies are inadequate, it hardly follows that "there are no fallacies in real life" (191).

There is, however, a way to eliminate fallacies: simply adopt a very strong principle of charity-a principle according to which "the best interpretation of an argu- ment is always that interpretation which makes it out to be the best argument", an argument "which contains no mistakes in reasoning" (197).

But strong charity is unreasonable: "it makes the presumption of rationality in others overbearing instead of having it function as one interpretive factor among others" (151). Moderate charity is preferable. It "directs us not to interpret others as having made implausible claims or faulty inferences unless there is good empirical reason to do so ... When relevant empirical evidence does not determine one or another interpretation and when moderate charity is indicated, we adopt that interpretation according to which the claims made are most plausible and the inferences most reasonable" (152).

Govier sees her principle of charity as a particular application of Grice's Principle of Cooperation. Its basis, she believes, is not to be found in ethics, prudence or epistemology, but in the purpose of argumentative discourse as such, which is "to rationally persuade a rationally critical audience" (149-50). We presume that when people appear to be engaged in argumentative discourse, they are "trying to put forward good reasons for claims that they believe" (150). This is a "rebuttable presumption" of course. But "[i]t gives a basis for moderate charity in the social practise of argument and its functional prerequisites" (150).

Charity as a principle of interpretation is one of several topics pertaining to the interpretation of discourse that a "fullfledged" theory of argument would cover. A second is the problem of missing premises, to which Govier devotes two chapters (see below, section 2). A third is the identification of arguments.

Arguments are different from explanations. One of the differences is epistemic: "in an argument certainty moves from the premises to the conclusion, whereas in an explanation it moves from the fact explained to the explanatory hypothesis" (162). A 
second difference is pragmatic: while the purpose of an argument is rationally to persuade an audience that a claim is true, the purpose of an explanation is "to show how or why something came to be as it is" (168). Given these differences, Stephen Thomas cannot be right that "the same logical criteria suffice to evaluate explanations and arguments" (173) (or rather, as Govier should have said, to evaluate explanations and arguments that are not explanations). But he is right that there are passages which, on the same interpretation, are both arguments and explanations. And it is true that "reasoning is used both in explanations and in arguments" (174). Thus we may want to agree with Thomas that "logicians who see themselves as appraising reasoning would do well to include explanation as well as argument within the scope of logic'" (175), and so within the scope of logic courses.

Logic courses often advertize themselves as courses in critical thinking. But their concern is with argument construction and argument analysis (in a broad sense that includes argument evaluation: 237) and critical thinking is not to be identified with either of these, though it includes both. It is not to be identified with argument construction because we often think critically without arguing. And it is not to be identified with argument analysis because (for one thing) there are products of critical thinking other than the analysis of an argument--e.g., a well-formulated question or an improved definition.

If there is more to critical thinking than argument construction and argument analysis, then logic courses cannot hope to cover all aspects of critical thinking. Neither can critical thinking tests (of the shortanswer machine-gradeable kind) hope to test all aspects of critical thinking. Their format makes this impossible. But then it is problematic to infer critical thinking ability from test results. Indeed there are further reasons why this is so, one of which is that the inference depends on the assump- tion that the keyed answers to the test questions are correct. But Govier does a "content analysis" of four commonly used tests (including the Watson-Glaser and Cornell Level $\mathrm{Z}$ tests) and finds in each case a significant percentage of contestable items. The tests are also flawed in other respects, she believes. If her criticisms are sound, then perhaps philosophers should bend their minds to improving the tests or inventing new ones. On the contrary, says Govier. Philosophers should use their critical thinking abilities "to resist those forces in society that demand a single number, obtainable after a 50-minute computer-scorable examination, to represent critical thinking ability" (268).

In her final chapter, entitled "The Social Epistemology of Argument', Govier questions the propriety of assessing arguments in isolation from their social and psychological contexts. There are, after all, circumstances in which an audience can base its acceptance of an arguer's claims on no evidence other than the sincerity of the arguer. In such circumstances, there is, and must be, a presumption that the arguer is trustworthy.

Indeed, there is always a presumption of trust in contexts of argument-a point Govier brings to bear on the tu quoque. This is an argument in which an arguer is accused of pragmatic inconsistency- "of failing to live up to his own expressed principles" (274). Such inconsistency obviously does not mean that the specific content of the argument in question is defective. But it does have a logical bearing on the argument's force. Thus, if an arguer, $X$, criticizes the practice of another person, $Y$, and if $X$ is accused of pragmatic inconsistency, the "burden of proof' shifts back to X, who "must show that he or she is not inconsistent, or suffer a loss of credibility" (276). Here Govier follows Douglas Walton. But she thinks that Walton has not explained why there is a shift in the burden of proof. The explanation, she suggests, can be found " in the disturbance of the general 
presumption of trust and sincerity underlying argument as a social institution' '(279).

There is a further area where "sociopersonal characteristics bear on the logical evaluation of arguments"' (280)-an area having to do not with arguers but with audiences. Govier pursues this point in the course of developing an audience-relative notion of argument cogency, which I shall discuss in Section 5.

In Sections 2-4 I shall discuss a selection of her views on three other topics.

\section{(2) The Problem of Missing Premises:}

Missing premises are unstated assumptions. But Govier argues persuasively that not all unstated assumptions are missing premises. Otherwise " every argument will be an enthymeme"' (92), for there are always some unstated assumptions upon which an argument depends. (An example would be a principle of inference, such as modus ponens: principles of inference cannot be written into arguments as unstated premises "on pain of a vicious infinite regress" (96).) Thus, if an argument has missing premises, these are a subset of its unstated assumptions.

Govier endorses Robert Ennis's view that the role of missing premises is to fill an "inference gap" in the stated argument. But people may disagree about whether a particular argument has an inference gap because they disagree about the theory of argument. For example, a reconstructive deductivist will see an inference gap in any argument which as stated is not deductively valid, but in some such arguments a pluralist will not.

Now if an argument is not deductively valid as stated, then, as noted above, a reconstructive deductivist will recommend that it be supplemented with one or more premises whose addition to the argument will make the argument deductively valid. Govier calls this the "unqualified deductivist policy on missing premises"' (85), and she doesn't like it. For one thing, the policy "licenses an infinite number of expansions for each argument"' (85). If one is flawed, this will not be "a definitive criticism of the original argument, for there are always an infinite number of alternative reconstructions remaining to be considered, and on purely deductivist grounds, these reconstructions are all of equal merit" (85). What is more, adding validating premises to an argument can be "excruciatingly pedantic" (87) and "extremely cumbersome" (88). Of course we can avoid this problem by adding as a missing premise 'the associated conditional'- a conditional statement whose antecedent is the argument's premise, or the conjunction of the argument's premises, and whose consequent is the conclusion. But this is "a critically redundant manoeuvre" (88), for the associated conditional "simply reiterates the original argument"' (86). By itself, then, reconstructive deductivism "cannot provide a complete and adequate policy on missing premises. It must be qualified so as to apply only to some arguments and supplemented so that not all deductivist reconstructions are of equal critical relevance"' (85).

Govier does not tell us, however, to what arguments reconstructive deductivism applies (or how to decide to what arguments it applies). For all we know, then, it may apply to arguments some of which it would be "excruciatingly pedantic" and "extremely cumbersome" to reconstruct as deductively valid arguments, if we agree to forego using the associated conditional as a missing premise. Thus Govier herself may not altogether avoid one of the problems she associates with the unqualified deductivist policy on missing premises.

Her own policy recommends the following procedure. If we interpret a discourse as containing an argument with stated premises and a stated conclusion, we classify the argument as being of some particular type, according to our theory of argument. We then make the "[l]ogical 
judgment that the stated argument is not inferentially sound as an example of that type" and the further logical judgment that it "would be inferentially sound if one of a candidate set of supplementary premises were added" (102). Next we make the " [l]ogical/epistemic/interpretive judgment that the argument is an enthymeme rather than a fallacy or non sequitur and that the gap identified should be filled" (102). Finally, we select one of the candidate premises to fill it. The selection should be "based both on epistemic (modest charity) considerations and on interpretive considerations-respect for the actual discourse and beliefs which the arguer held or would have been likely to hold" (102).

An argument with a stated conclusion is not an enthymeme, then, if, as stated, it has a sound inference. But when is an inference sound? Govier quotes Lambert and Ulrich as saying that " $[\mathrm{a}] \mathrm{n}$ inference is sound just in case the argument representing it is sound" and that "[a]n argument is sound if and only if (1) it is valid and (2) all its premises are true" (22). Govier's own conditions for argument soundness are, needless to say, different. In her textbook ${ }^{2}$ she takes the position that an argument is sound if and only if its premises are acceptable and properly connected to the conclusion and that premises are properly connected to a conclusion if and only if they are positively relevant to the conclusion and provide adequate grounds for $\mathrm{it}^{3}$. But she does not say, either in her textbook or in the present work, when an inference is sound. In particular, then, she does not say that

(i) an inference is sound just in case the premises in the argument representing it are properly connected to the conclusion

or, as Lambert and Ulrich do, that

(ii) an inference is sound just in case the argument representing it is sound.

But there is evidence that if she subscribes either to (i) or to (ii), it is (i) to which she subscribes, not (ii). For she distinguishes between: "the appraisal of reasoning" and "the appraisal of premises" (232); "substantive errors" and "reasoning errors" (235); "implausible claims" and "faulty inferences" (152); plausible claims and reasonable inferences (152); " $[\mathrm{t}$ ]he acceptability of premises" and "inferential relationships" (281). On the other hand, in the present work she treats question-begging as an inference flaw, while in her textbook she treats it as a premise flaw. But when she claims in the present work that an argument which begs the question is "inferentially flawed" (282), she refers us, in a footnote, to her textbook treatment of questionbegging ${ }^{4}$. And this is some reason to think that for her premise acceptability is a condition of inferential soundness, hence that if she subscribes either to (i) or to (ii), it is (ii) to which she subscribes, not (i). However in my judgment the evidence that she subscribes to (i) outweighs the evidence that she subscribes to (ii), assuming that she subscribes either to (i) or to (ii).

But there is a further problem. If Govier subscribes either to (i) or to (ii), she believes that there are conditions for inferential soundness that apply to arguments of all types. If she subscribes to (i), then (according to her textbook) these are relevance (the requirement that an argument's premises be positively relevant to the conclusion) and adequacy (the requirement that an argument's premises provide adequate grounds for the conclusion), while if she subscribes to (ii) her conditions for inferential soundness are relevance, adequacy and acceptability (the requirement that an argument's premises be acceptable). But in presenting her position on missing premises, Govier makes two points either of which might cause us to doubt that she thinks there are conditions for inferential soundness applicable to arguments of all types. One is that an argument has a missing premise only if it is not inferentially sound as an example of a certain argument-type. The other 
is that to decide whether a particular argument has a missing premise, we need a concept of complete argument which we can properly apply to it. We need such a concept, I take it, in order to decide whether the argument is inferentially sound as an example of the type we judge it to exemplify. But if so, then we might suppose that in Govier's view there are different "concepts" of inferential soundness for arguments of different types. And we might regard the first point as evidence to the same effect. However immediately after making the second point, Govier adds that the required concept "need not be applicable to all arguments" (102). But this is to allow that it may be so applicable-that there may be a "concept" of inferential soundness applicable across arguments of all types. The first point also permits this view. Both points leave Govier free to hold that there are conditions for inferential soundness that apply to arguments of all types, but that what is required for an argument to satisfy one or more of these conditions varies with different argument-types. Thus suppose that the conditions are relevance and adequacy. Consistently with holding that these conditions apply to arguments of all types, Govier could hold that, for example, what is required for an enumerative induction to satisfy the adequacy condition is different from what is required for an analogical argument to do so. And in fact she does hold precisely this position in her textbook. Thus she holds that an enumerative induction satisfies the adequacy condition if and only if the sample is large enough and representative enough to be a guide to the population (PSA 279) and that an analogical argument satisfies the adequacy condition if and only if there are no decisive negatively relevant differences between the cases compared (PSA 224, 231).

I propose to assume that in Govier's view there are indeed conditions of inferential soundness for arguments of all types, and that these are relevance and adequacy. Thus I propose to assume that she holds position (i), not (ii). If in fact she holds position (ii), this will not much matter because my discussion could easily be revised accordingly.

An argument with a stated conclusion is an enthymeme, according to Govier, only if it has an unsound inference. But some arguments with unsound inferences are fallacies and a fallacy is not an enthymeme. How then do we decide whether an argument with a stated conclusion and an unsound inference is a fallacy or an enthymeme? In a footnote Govier remarks that this is a "tough issue" (104). Indeed it is; unfortunately it is one she does not discuss. It would have been useful had she provided examples of arguments of several different types, all of them inferentially unsound but not all of them fallacies, and explained in each case how it is decided that the argument is a fallacy or that it is not. But she does not do this.

She does, however, consider seven passages containing arguments with a view to deciding whether the arguments have missing premises and, if so, what they are. This happens in the course of a skilfully constructed dialogue between characters named Charmides and Lysis, who have different policies on missing premises. That of Charmides is more permissive than that of Lysis, for Charmides favours adding premises in cases in which Lysis does not. It is Lysis who speaks for Govier. But Lysis makes some puzzling remarks. Thus in one case he judges that the argument has no inference gap because " $[t]$ he direction of the inference is quite clear" (119). Now for the "direction" of an inference to be clear, it is surely enough that it be clear what the argument's conclusion is and what its stated premises are. But if so, then an argument may have an inference gap, at least in the sense of the phrase that Govier earlier invoked to explain her position on missing premises, even if the "direction" of the inference is clear. In another case, Lysis says that there is "no inference gap in the sense that we cannot understand how we are sup- 
posed to connect the stated premises with the conclusion"' (115). But an argument with a stated conclusion is an enthymeme (Govier has told us) only if there is an inference gap in the sense that the argument has a flawed inference. And this is a different sense of the phrase. For an argument may have a flawed inference even if we can understand "how we are supposed to connect the stated premises with the conclusion'. For example, we may see that the argument "is supposed to work as a syllogism", or that it is "supposed to work as a formally valid propositional argument", (95), yet also see that, as stated, it has a flawed inference. Or so Govier leads us to believe when stating her views on missing premises (95). Thus when stating those views she uses the phrase 'inference gap' in a sense different from the one (or two) in which she allows Lysis to use it. And this is puzzling, not to say confusing, if Lysis's position on missing premises is supposed to be the same as Govier's. One more example. Speaking of an analogical argument, Lysis says this: "Whether the analogy is good or not, seems to be a moot point, but in any event there is no need to insert a supplementary premise since the intended direction of the inference is entirely clear" (119). But according to Govier's position on missing premises, whether there is a need to insert a supplementary premise in an argument depends in part on whether the argument has a flawed inference. But if relevance is one of Govier's conditions for inferential soundness, then whether an analogical argument has a flawed inference depends for her precisely on whether the analogy on which the argument is based is good or not, because it depends on whether the cases compared are relevantly similar (PSA 224).

\section{(3) Argument by A Priori Analogy:}

This for Govier is one of two kinds of analogical reasoning, the other being argu- ment by inductive analogy. An a priori (or logical) analogy is an argument by analogy which issues in a decision and in which "it does not matter whether the instance used as a basis for comparison [which in PSA Govier calls the analogue] is actual or hypothetical' (58). ${ }^{5}$ Thus if I decide that abortion is morally wrong on the ground that it is relevantly similar to infanticide, which I regard as a paradigm case of moral wrongdoing, I reason by a priori analogy. An inductive analogy, by contrast, is " [a]n argument by analogy in which the conclusion is predicted on the basis of experience with the analogue"' (PSA 242), which must be a real case, not a hypothetical one. The kind of analogical argument in which Govier is mainly interested in the present work is argument by a priori analogy, for she believes that analogical reasoning of this sort has been ignored by many supporters of deductivist and positivist theories of argument and, indeed, that such theories cannot accommodate a priori analogies without paying an unreasonable price. But she has some things to say about analogical arguments in general. For example, she believes that "any analogy" has the following form (59):

(A) (1) Case $x$ has features $a, b, c$.

(2) Case y also has features a, b, c.

(3) Case $x$ is of type e.

Therefore,

(4) Case $y$ is of type e.

An argument of this form is not deductively valid. A reconstructive deductivist would therefore favour recasting it as a deductively valid argument. And Govier acknowledges that this can readily be done, by adding a suitable general premise (59). Thus:

(B) (1) Case $x$ has features $a, b, c$.

(2) Case y has features a, b, c.

(3) Case $x$ is of type e.

Missing Premise: All things which have features a, b, c, are of type e.

Therefore,

(4) Case $y$ is of type e. 
Moreover Govier's criticisms of reconstructive deductivism leave her free to hold that it is perfectly appropriate to recast an analogical argument in this fashion. For her criticisms are directed at an unqualified deductivist policy on missing premises. She does not deny that there are some arguments which as stated are not deductively valid but which it is appropriate to reconstruct as deductively valid arguments. Consistently with her criticisms of reconstructive deductivism, then, she could maintain that these include analogical arguments and that it is appropriate to recast such arguments in form (B). As it happens, however, this is not her view. She thinks it is a mistake to recast an analogical argument in form (B). For one thing, the addition of the missing premise makes the premises about case (x) redundant "as far as the logic of the inference is concerned" (60). Thus to add the premise is not to recast the analogy but to destroy it. Admittedly one might take the view that case ( $\mathrm{x}$ ) fulfils a psychological function in the argument by serving "to remind us of the general truth under which case (y) is subsumed" (60). But this still leaves the result that the analogy plays no logical role in the argument; it is merely "a psychological crutch" (60). Moreover -and this is an "even more basic problem"- the required generalization is "typically not known" (60). Thus to supplement analogical arguments with general premises is usually "to weaken them epistemically" (60). Usually it is also "to distort their logical character", for usually they involve "the direct comparison of cases without appeal to an intermediate generalization" (60).

This last point is indisputably correct as a matter of empirical fact. But the relevant question about the "logical character" of analogical arguments is whether they require general premises if their inferences are to go through. This is apparent from Govier's position on missing premises. But when Govier discusses analogical arguments (in Chapter 4) she has not yet explained her position on missing premises: she does this in Chapter 5. Thus, unless we read Chapter 5 before Chapter 4 , when we first read Chapter 4 we do not know that for Govier the role of a missing premise is to fill an inference gap. Had we known that this was her view, we might have expected her to argue that an analogical argument may have a sound inference even in the absence of a general premise. This would have been for her to argue that an analogical argument which lacks a general premise may satisfy her conditions for inferential soundness. But the argument would not have impressed a deductivist (reconstructive or literal). For the argument would not have been that an analogical argument may be deductively valid and hence inferentially sound in the absence of a general premise. It would have been that an analogical argument which is not deductively valid may be inferentially sound in the absence of a general premise: for Govier thinks that analogical arguments are not deductively valid but that they may nevertheless be inferentially sound. And this the deductivist will deny. Of course as a pluralist Govier thinks that deductivism is mistaken in holding that no argument is inferentially sound unless it is deductively valid. But she still has to contend with a qualified deductivist who holds, as she does herself, that some arguments are not inferentially sound unless they are deductively valid and who also holds, as she does not, that analogical arguments are a case in point. Against a qualified deductivist of this persuasion Govier would beg the question were she to argue as follows: It is possible for the premises of an analogical argument to provide adequate support for (and hence ${ }^{6}$ be positively relevant to) the conclusion. But no analogical argument is deductively valid. Thus it is possible for an analogical argument to be inferentially sound despite not being deductively valid. It is not questionbegging, however, to argue that a reconstructive-deductivist approach to analogical arguments is mistaken because 
to recast an analogical argument so that it is deductively valid by adding to it a suitable generalization as a missing premise is to make the analogy logically redundant and weaken the argument epistemically. Which, as we have seen, is precisely what Govier does argue.

More specifically, she argues that it is a mistake to recast an analogical argument of form (A) as a deductively valid argument by adding to it a generalization as a missing premise. Of course this does not commit her to the view that any argument of this form is inferentially sound. To decide whether a particular analogical argument is inferentially sound, we need a concept of complete analogical argument. For to decide whether a particular argument of no matter what type is inferentially sound we need a concept of complete argument of that type. (At any rate, to decide whether the argument has a missing premise we need such a concept, and I am assuming that the reason we need it, in Govier's view, is to decide whether the argument as stated is inferentially sound.) But Govier also cites cases in which "we apply a familiar model to generate a missing premise" (95). Thus I take it she would say that to have a concept of complete argument of some type may be to have a model. Now for the purposes of this discussion I wish to stipulate that a model of complete argument of some type would be an argument-form such that any argument of that form is inferentially sound by what I am assuming to be Govier's criteria of inferential soundness. And I wish to take up two questions. The first is whether (A) is a model of complete argument by analogy. The second is whether there is a deductive model of complete argument by a priori analogy that is not vulnerable to Govier's criticisms of model (B), as I shall call it.

If an analogical argument specifies similarities between the cases it compares, then, Govier will say, the stated argument satisfies the relevance condition if and only if the specified similarities are relevant
(PSA 224). An analogical argument of form (A) specifies similarities between the cases it compares. But they may not be relevant. Hence (A) is not a model of complete analogical argument.

It follows that (A) is not a model of complete argument by a priori analogy. But consider

(C) (1) Case $x$ has features $a, b, c$.

(1a) Case $x$ is of type e in virtue of having features $a, b, c$.

(2) Case y has features a, b, c.

Therefore,

(4) Case y is of type e.

The truth of (1a) would be sufficient for the stated similarities between the two cases to be relevant, assuming they are real. For if cases $(\mathrm{x})$ and $(\mathrm{y})$ have features $\mathrm{a}, \mathrm{b}, \mathrm{c}$ in common, these are relevant similarities if case ( $\mathrm{x}$ ) is (treated as being) of type e in virtue of having those features. (The reason why the similarities are relevant in this event is that consistency requires that if case ( $x$ ) is treated as being of type $e$ in virtue of having features a, b, c, then, other things equal, case (y) must be treated similarly given that it too has those features. ${ }^{7}$ ) An a priori analogy of form (C), then, satisfies the relevance condition. But it may not satisfy the adequacy condition, for there may be a decisive negatively relevant difference between the cases compared. Thus (C) is not a model of complete argument by a priori analogy.

But let us continue. Govier remarks that "[i]f two cases are deemed to possess feature e in virtue of other features $a, b$, $c$ then that implies that the features $a, b$, $c$, function to establish $\mathrm{e}$-other things being equal" (64). Quite so. Indeed, this is implied if one case is deemed to possess feature e in virtue of other features $a, b$, c. Accordingly, (1a) implies a generalization to the effect that

(5) All things which have features $a, b$, $c$ are of type $\mathrm{e}-$ other things equal.

For if one holds that features a, b, c of case 
(x) make (x) a token of type e, then, in all consistency, one must hold that if any case whatsoever has these features, then it is a token of type e, other things equal. To argue by a priori analogy, then, is to be logically committed to (5) or to a generalization logically equivalent thereto. Moreover, we can include (5) as a premise in a model of complete argument by a priori analogy without making logically redundant a premise referring to the analogue (in Govier's sense of the word). Thus:

(D) (1a) Case $\mathrm{x}$ is of type $\mathrm{e}$ in virtue of having features $a, b, c$.

Therefore,

(5) All things which have features a, b, $c$ are of type e, other things equal.

Therefore,

(6) If case y has features a, b, c, it is of type e, other things equal.

(2) Case y has features a, b, c.

(7) Other things are equal. Therefore,

(4) Case $y$ is of type e.

There are three inferences here and each is deductively valid. Govier holds that a deductively valid argument satisfies the relevance and adequacy conditions (PSA 64). Hence (D) is a model of complete argument by a priori analogy - a deductive model.

Note that premise (7) is the claim that other things are equal in case $(y)$. It is not a generalization to the effect that other things are equal in any case with features $a, b, c$. If (7) were a generalization to this effect, premise (6) in (D) would be logically redundant, for then (5) and (7) would jointly imply

(8) All things which have features a, b, c are of type e,

and (4) would follow just from (8) and (2) jointly.

But if (7) is not a generalization, (5) is. Unlike the generalization in model (B), however, (5) does not make a premise about the analogue (case $(\mathrm{x})$ ) logically redundant.
Case ( $\mathrm{x}$ ) does not have the merely psychological function of serving to "remind" us of (5). Rather, it has the logical function of providing support for (5). Moreover if we endorse model (D) we need not take the view that (5) is known prior to inspection of case (x). On the contrary, we may hold that it is precisely inspection of case (x) that makes it known to us. (This would be an instance of knowing the universal "in and through the particular"' (74)-a phenomenon to which Govier draws our attention later in Chapter 4.) At any rate, (5) is no less epistemically acceptable than (la) - the premise about case ( $x$ ) by which it is implied. And if we have an argument by a priori analogy whose conclusion is that case $(y)$ is of type e and whose premises assert just that cases ( $x$ ) and (y) have features a, b, c and that case ( $\mathrm{x}$ ) is of type $\mathrm{e}$, the arguer would accept the premise that case $(x)$ is of type e on the ground that $(x)$ has features a, b, c. But in accepting the premise for this reason, the arguer would assume (1a) or would make a logically equivalent assumption. But then adding (1a) to the argument as a premise would not weaken the argument epistemically. Nor, then, would adding (5) as an intermediate conclusion implied by (la). Thus Govier cannot legitimately object that to reconstruct the argument according to model (D) would be to weaken it epistemically by adding (5) to it as a missing premise. For in (D) (5) is not only a premise but also a conclusion implied by (1a). I conclude that the grounds on which Govier objects to model (B) do not show that it is a mistake to endorse model (D) as a (deductive) model of complete argument by a priori analogy.

But there is more to be said about (D). The force of (7) is that there is no reason to decide that case (y) is not of type e which overrides the reason given for deciding that it is of type e, namely (2). Now to ask why (2) counts as an (overridable) reason for deciding that case $(y)$ is of type $e$ is in effect to ask why (6) is true, if it is. One answer is that if (6) and (5) are true, then 
(6) is true because (5) is true. But another is that if (6) and (1a) are true, then (6) is true because (1a) is true. For (6) is implied by (la). Accordingly, we do not need (5) in order to derive (6), and this is an objection to model (D). Removing (5) from (D) would give us

(E) (la)Case $x$ is of type e in virtue of having features $\mathrm{a}, \mathrm{b}, \mathrm{c}$.

Therefore,

(6) If case $y$ has features $a, b, c$, it is of type e, other things equal.

(2) Case y has features a, b, c.

(7) Other things are equal.

Therefore,

(4) Case y is of type e.

Like (D), this is a deductive model of complete argument by a priori analogy. But, unlike (D), it does not include a general premise, and this is sufficient to ensure that, despite being deductive, it is not subject to the specific criticisms Govier makes of model (B).

It is most certainly not my claim, however, that an argument by a priori analogy must be reconstructed according to a deductive model if it is to be inferentially sound (by the relevance and adequacy criteria). On the contrary. It may be inferentially sound (by those criteria), if, for example, it is reconstructed according to model (A). To grant this is of course to grant that an analogical argument of form (A) may be inferentially sound (by those criteria) and hence (if its conclusion is stated) that it is not what Govier would call an enthymeme.

Nevertheless it is noteworthy that there are deductive models of complete argument by a priori analogy that are not vulnerable to her criticisms of model (B). For this means that she has not succeeded in showing that neither reconstructive deductivism nor positivism can accommodate a priori analogies without paying an unreasonable price. For all she has shown, both can.

\section{(4) Conductive Argument:}

This is a further kind of argument that in Govier's view neither reconstructive deductivism nor positivism can reasonably accommodate. My interest here is in her reasons for thinking that this is so. I begin with her account of a conductive argumerit.

A conductive argument with two or more premises is a convergent argument. (But not all convergent arguments are conductive.) If the premises "count for the conclusion" (70), they do so by being separately and nonconclusively relevant to it: separately, "in the sense that if one were false, the others would remain unaffected" (69); nonconclusively, because none of them entails the conclusion, nor do they jointly entail it. A conductive argument need not be a multi-premise argument, however. In "the limiting case" (70) there is only one premise, but "other factors, not mentioned, could have been mentioned to count as well" (67-8). Whether the premise(s) of a conductive argument is (are) relevant to the conclusion " is a conceptual, normative, or 'criterial' issue" (70): hence conductive reasoning is nonempirical. Finally, conductive arguments "naturally admit counterconsiderations as part of the argument" (69)-considerations that count against the conclusion but which, in the arguer's estimation, are outweighed by the premise (in the limiting case) or by the premises taken together (in the standard case).

Because conductive reasoning is both nonconclusive and nonempirical, it is for Govier neither deductive nor (classically) inductive. A reconstructive deductivist, of course, will want to reconstruct conductive arguments as deductively valid ones, and so might a positivist. But Govier believes that Carl Wellman has shown that this approach to conductive arguments is wrongheaded. Thus consider "such an argument as "you should return the book because you promised to do so" "(73). If we try to turn this into a deductively valid argument, we will need one or more fur- 
ther premises. But:

Possible additional premises are either false, unverifiable independently of a judgment about the individual case, or impossible to formulate in advance. That you should always keep promises is false; that you should always keep promises other things being equal is unverifiable independently of a judgment about the case at issue; that you should always keep promises in circumstances of type (abc) is impossible to formulate in advance. The enthymeme approach, here as so often, makes an inference watertight at the cost of introducing an unknowable premise (73).

(In rejecting this approach, Govier does not, I take it, intend to imply that no conductive argument is an enthymeme. ${ }^{8}$ Rather her point is simply that it is a mistake to interpret conductive arguments as elliptical deductive arguments, hence as arguments which it is appropriate to reconstruct as deductively valid arguments. Accordingly, by 'the enthymeme approach to conductive arguments' I shall here mean the view that conductive arguments are elliptical deductive arguments.)

In Govier's judgment, a conductive inference is not "watertight". But she would allow that it may be sound, for she believes that a conductive argument may be a good argument. To be good, in her eyes, a conductive argument must satisfy the relevance and adequacy conditions (70, PSA 250), which I shall continue to assume are her conditions for inferential soundness. A conductive argument, like an argument of any other kind, satisfies the relevance condition if and only if its premise(s) is (are) positively relevant to its conclusion. It satisfies the adequacy condition if and only if its premise outweighs (or its premises taken together outweigh) any stated or unstated counterconsiderations, taken together, that are negatively relevant to its conclusion.

Is the inference in the "book" argument sound (by these criteria)? Not if there are unmentioned factors negatively relevant to the conclusion that would outweigh the premise if measured against it. But we don't know whether there are such factors, because we don't know whether there are unmentioned factors that are negatively relevant to the conclusion: we don't have the background information required to answer this question. Thus we don't know whether the argument satisfies the adequacy condition. And so we don't know whether the inference is sound.

Suppose, however, that we reconstruct the argument as a deductively valid argument, as follows:

(1) You should always keep your promises, other things equal.

(2) You promised to return the book.

(3) Other things are equal.

Therefore,

(4) You should return the book.

We know that this argument has a sound inference (by the relevance and adequacy criteria-assuming, with Govier, that a deductively valid argument satisfies those criteria: PSA 64). But, for want of background information, we do not know whether premise (3) is true. When it comes to assessing the argument, then, it matters not whether we reconstruct it as a deductively valid argument (with at least one "unknowable premise") or take it to be an argument-that-is-not-an-elliptical-deductiveargument (with an inference whose soundness is undecidable). We are in the same boat in either case-a point Govier seems not to have noticed.

But now assume that we do have background information enabling us to decide whether the argument, as stated, satisfies the adequacy condition and hence ${ }^{9}$ has a sound inference. The same information will enable us to assess premise (3) in our deductively-valid reconstruction of the argument. For other things are equal just in case there are no unmentioned factors negatively relevant to the conclusion that outweigh premise (2). And whether there are such factors is just what we must know to decide whether the stated argument has a sound inference. So when it comes to 
assessing the argument, it still makes no difference whether we reconstruct it as the deductively valid argument given above or take it to be an argument that is not an elliptical deductive argument. (I assume that premise (1) in the reconstruction is "knowable",.10)

But Govier is not finished. She claims that additional premises proposed to make a conductive argument deductively valid "may distort the original argument, which is typically not put forward as being conclusive"' (73). This is a slightly surprising remark, given her pronouncement in an earlier chapter that " $[w]$ hen people present arguments, it is very often quite unclear whether logical entailment or merely some less tight support is "claimed", (39). More importantly, Govier is here implicitly admitting that a conductive argument may be put forward as being conclusive, and that in such a case additional premises proposed to make the argument deductively valid (if it is not deductively valid as stated) would not distort the argument. But a conductive argument that is not put forward as being conclusive may be put forward as being nonconclusive; and in such a case, I would agree, to add to the argument premises sufficient to make the argument deductively valid would be to distort the argument and would therefore be a mistake.

Suppose, however, that a conductive argument is not put forward as being conclusive or as being nonconclusive. An example, as far as we can tell, is the "book" argument, which employs a logical indicator ("because") that is neutral as between a claim of conclusiveness and a claim of nonconclusiveness. If we add premises sufficient to make the argument deductively valid, the result is an argument we put forward as being conclusive (or so I shall assume). Strictly speaking, this is to distort the original argument. But the distortion is innocuous, at least if the deductively valid version of the argument is the one given above. For the claim of conclusiveness that we assumedly make if we reconstruct the argument in that way is true. Admittedly, if we reconstruct the argument in that way we are committed to the further claim that 'you promised' is a reason to return the book that is not overridden by other considerations. But Govier can hardly regard this as a weakness of the reconstructed argument. For the inference of the original argument is sound (by what I am assuming are her criteria) only if precisely the same claim is true; for the inference is sound only if there are no unmentioned factors negatively relevant to the conclusion that outweigh the premise that you promised to return the book.

Govier has a further critical point, which is that when a deductivist reconstruction is given of a conductive argument with more than one explicit premise, "one argument is turned into several"' (73). Really? Suppose that we have a conductive argument with explicit premises $\mathrm{P}$ and $\mathrm{Q}$ and conclusion $\mathrm{C}$. The structure of the argument is this:

(a)

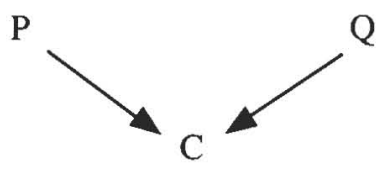

Now suppose that we add validating premises $\mathrm{R}$ and $\mathrm{S}$, giving us

(b)

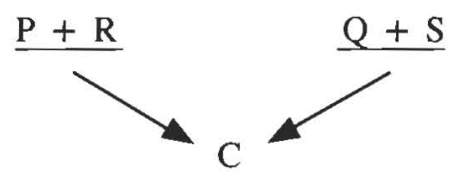

If (a) is one argument, not two, why isn't (b)? Consider the following reply. Since (a) is a conductive argument, $\mathrm{P}$ and $\mathrm{Q}$ strengthen each other: their "cumulative force" (68) is greater than the force of either by itself (assuming that both are positively relevant to $\mathrm{C}$ ). But because the inferences in (b) are each conclusive, the premise-sets ( $\mathrm{P}$ and $\mathrm{R}$ ) and ( $\mathrm{Q}$ and $\mathrm{S}$ ) do not strengthen each other: their cumulative force is not greater than the force of either set by itself. Thus (b) is really two (conclusive) arguments (for the same conclusion) while 
(a) is one (non-conclusive) argument. But this is a non sequitur. What it shows is that (b) is not a conductive argument. It does not show that it is two arguments. In any case, it appears that Govier is not at liberty to argue that because the inferences in (b) are each conclusive, (b) is not one argument but two. For when she makes the point that not all convergent arguments are conductive, she says: "It is possible to offer an argument which exemplifies the convergent support pattern, but in which there are several premises, each of which, taken alone, deductively entails the conclusion", (70). This would be one argument, not several arguments with the same conclusion. In all consistency, then, Govier must say that (b) is a single argument. But then she cannot without inconsistency object that adding to a multi-premise conductive argument premises sufficient to make the argument deductively valid turns one argument into several.

In sum. A reconstructive deductivist will take the enthymeme approach to conductive arguments, and a positivist might do the same. Govier has not demonstrated that this approach is always mistaken. Nor has she demonstrated that we will always be in a better position to assess conductive arguments if we reject the enthymeme approach in favour of her own.

\section{(5) Argument Cogency:}

A cogent argument for Govier has acceptable premises. Acceptable premises need not be true. Thus for Govier a cogent argument need not have true premises. On a rival view, a cogent argument is a sound argument as traditionally conceived-a deductively valid argument with true premises. One problem Govier finds with this view is that "if we stipulate that people should be convinced only by those arguments that have true premises, we would in effect be stipulating that in many times and places, people should not be con- vinced by arguments at all" (280). This being so, "standards of argument appraisal that are intended to give real practical guidance will have to move from truth of premises to acceptability" (280). Now "[a]cceptability is a relative notion"' (280): an argument's premises must be acceptable to the audience addressed. Moreover, Govier claims (for reasons I give below) that there is "a degree of audience relativity even in inferential relationships" (281). If so, and if an argument's premises must be acceptable to the audience addressed, then the notion of argument cogency is audiencerelative: a cogent argument is cogent for an audience. Not, however, for a universal audience, because "discourse that is literally designed for everyone does not exist" (281). Rather, for a particular audience. Cogent "arguments hold for those-in-acontext, and not necessarily for all in all contexts"' (289).

"An audience is given cogent argumentation if it is given argumentation in which premises are acceptable to it and are connected to the conclusion in a.way that is appropriate"' (280). This is not to say that an argument is cogent if "some audience in fact has found it convincing" (284). Adopting an audience-relative notion of argument cogency does not require us to "reject the distinction between what is in fact taken as cogent by an audience and what that audience ought to take as cogent" (284). For " $[\mathrm{t}]$ here are norms here, regarding acceptability of premises and appropriacy of their link with the conclusion" (280). But these norms incorporate some audience-relativity. Thus "an argument is cogent for an audience if, according to standards that audience would deem on reflection to be relevant, the premises are acceptable and in the appropriate way sufficient to support the conclusion"' (287).

In her textbook Govier takes the view that acceptability, relevance and adequacy are the "basic conditions of argument cogency" (PSA 63). This view is fully compatible with her present view that argument 
cogency is audience-relative. For an argument that is cogent for an audience satisfies her textbook conditions of argument cogency. Thus an argument cogent for an audience has acceptable premises-premises acceptable to the audience addressed. And it satisfies the adequacy condition, for its premises are (in the appropriate way) sufficient to support the conclusion. And adequacy entails relevance (PSA 62). What Govier's audience-relative account of argument cogency adds to her textbook treatment of the subject is that whether an argument satisfies her textbook conditions of cogency depends on whether it satisfies audience-relative standards-standards for deciding whether "the premises are acceptable and in the appropriate way sufficient to support the conclusion"'.

There is nothing to stop Govier from claiming that there are certain standards for deciding these questions which any audience interested in the appraisal of arguments should accept. In particular, then, there is nothing to stop her from claiming that there are certain standards of premise acceptability which any such audience should accept. And in effect she does precisely this in her textbook, where she lists a set of conditions the satisfaction of any one of which is (she claims) sufficient for a premise to be acceptable and another set the satisfaction of any one of which is (she claims) sufficient for the premises of an argument to be unacceptable (PSA 85, 92). But to be consistent with her audience-relative account of argument cogency, she must admit that if on reflection the readers of her textbook would deem relevant different conditions (standards) of premise acceptability, then whether an argument addressed to an audience of those readers has acceptable premises depends on whether its premises are acceptable to that audience according to those conditions (standards), not hers.

Consistency also requires Govier to refrain from claiming absolute cogency for her "argument that arguments hold for those-in-a-context, and not necessarily for all in all contexts" (289). And she does refrain from claiming this. She claims only that her argument is cogent for its intended audience, an audience which includes you and me.

Is it? I think it has certain merits and certain defects. Undoubtedly it is a merit of the argument that it allows, indeed insists, that even if an audience accepts the premises of an argument addressed to it, the premises are not acceptable to it if they fail to satisfy certain standards. "Acceptability is not acceptance"' (284). True enough. But there is a problem with the view that the standards that premises are required to satisfy to be acceptable to a particular audience are ones that the audience "would deem on reflection to be relevant". The problem is that such standards might be unreasonable. For example. Let the audience believe that a premise is acceptable if endorsed by the local religious authority and unacceptable if repudiated thereby. The audience's veneration of religious authority might be so deep that its members would not abandon this standard of premise acceptability however long or earnestly they reflected. But the standard is plainly unreasonable. This is undisturbing if we are allowed to say that premises acceptable to an audience may be unacceptable in themselves. But on Govier's audience-relative account of argument cogency we are not allowed to say this.

That account is a "contextual account" (286). Govier's contextualism is similar in substance, not just in name, to epistemic contextualism, which, as Paul Moser roughly defines it, is the view that "the foundations of justification are those beliefs that a certain community of believers takes for granted, or accepts without any reasons." 11 Moser objects that "what certain people happen to believe is irrelevant, from an epistemic point of view, to what there is good reason for one to believe true" '. ${ }^{12}$ Now perhaps we should not go so far as to say, analogously, that if an audience would on reflection deem (believe) 
a particular standard relevant to the appraisal of premises (or inferences), this is irrelevant, from an epistemic point of view, to whether there is good reason for one to believe it true that the standard is relevant. But we should certainly say that if on reflection the audience would believe the standard relevant, this would not ensure that the reasons for which it did so were good reasons to believe it true that the standard is relevant. Of course this could be ensured by stipulating that the audience conform to some appropriate description, such as that of having ideally reasonable members. But a stipulation of this sort is foreign to Govier's contextualism.

So is the stipulation that

(a) "people should be convinced only by those arguments that have true premises" (280).

Govier assumes that there is a connection between holding that a cogent argument is a sound argument as traditionally conceived, hence an argument with true premises, and making this stipulation. But it is clear that we need not so stipulate if we hold that a cogent argument has true premises. We are free to stipulate instead that

(b) people should be convinced only by those arguments whose premises they are epistemically justified in believing true.

Of course (b) entails (a) if epistemic justification entails truth; and (b) is intensionally equivalent to (a) if epistemic justification and truth are identical. But neither view is plausible-at least not on the absolute conception of truth, which states that it is true that $p$ if and only if $p .{ }^{13}$ (And it is this conception of truth that Govier associates with the traditional view of a sound argument). For, as Moser nicely puts it, epistemic justification is "perspectival"' 14 in a way that truth so conceived is not: a belief that is epistemically justified for you may not be for me. But if epistemic justification neither entails nor is identical with truth, then an epistemically justified belief may be false. Thus stipulation (b) is not intensionally equivalent to, and does not entail, stipulation (a).

Govier objects to (a) on the ground that to make this stipulation is in effect to stipulate that

(c) "in many times and places, people should not be convinced by arguments at all', (280)

because (presumably) in many times and places arguments have premises one or more of which are false. But stipulation (b), unlike (a), permits the view that people may rightly be convinced by an argument whose premises are false. Thus, to stipulate (b) is not in effect to stipulate (c). Now if holding that a cogent argument has true premises allows us to make stipulation (b), and not (a) or (c), then we do not need "to move from truth of premises to acceptability' ' in order to avoid the admittedly unwelcome stipulation that in many times and places people should not be convinced by arguments at all. Thus Govier does not persuade me that it is a mistake to believe that a cogent argument has true premises.

She does persuade me, however, that there is a degree of audience-relativity in certain inferential relationships. This seems clearly so in the case of arguments of types recognized by her theory of argument that as stated are not (or not usually) deductively valid. As she puts it: "The degree of certainty required for the conclusion, and hence the standards for evaluating inductive or analogical inferences, may vary depending on the context. The seriousness with which countervailing factors in conductive arguments are considered may be similarly affected"' (282). But Govier believes that audience-relativity enters into inferential relationships even in arguments that are deductively valid. In support of this belief she makes two points. One is that an argument which commits the fallacy of begging the question is deductively valid " yet inferentially flawed, because the audience 
cannot rationally move from acceptance of the premises to acceptance of the conclusion" (282). Now this is tantamount to saying that the inference is flawed because the argument fails on the adequacy condition, as that condition is elucidated in Govier's textbook (PSA 62). But there Govier says unequivocally that any deductively valid argument satisfies the adequacy condition (PSA 64)-including, then, one that begs the question.

Her second point in support of the claim that audience-relativity enters into inferential relationships in deductively valid arguments is meant also to illustrate a claim about all arguments, namely that, to be cogent, "argumentation must be intelligible to the persons to whom it is addressed" (281). The point is that if the premises of an argument entailed the conclusion but could be seen to do so "only by one world super-expert, the argument would not be cogent for most audiences, even though it was valid"' (282). Does Govier think, then, that the argument's inference would be unsound (for most audiences)? I would prefer to say that a deductively valid argument has a sound inference regardless of whether the inference is understood by the audience addressed. But I think Govier is right that if the inference is not understood by the audience, the argument is not cogent for that audience. In concluding, I will suggest (but not argue for) an interpretation of what it is for an argument to be cogent for an audience on which these two views are compatible, namely this: an argument is cogent for an audience if and only if the audience is epistemically justified in believing that the argument is cogent, a cogent argument being one with a sound inference and sound premises. Four brief points:

(1) Govier is surely right that an inference need not be valid to be sound. If so, then a cogent argument (as just defined) need not be a sound argument as traditionally conceived.

(2) There may be good reasons for requiring a cogent argument to have true premises. (I shall not take a position on this.) If there are, then sound premises are true premises. But even if truth is a virtue of sound premises, it is surely not their only virtue: another-as Govier's textbook position on acceptable premises would encourage us to believe-is that of not being question-begging.

(3) If sound premises are true premises, then an argument is cogent for an audience only if the audience is epistemically justified in believing its premises true. And the audience may be so justified (as Govier would agree) even if the premises are false.

(4) If an argument's inference is not intelligible to the argument's audience, the audience is not epistemically justified in believing that the inference is sound. Yet it may be that the inference is valid (and if it is that it is sound). Thus Govier's view is accommodated that an argument's inference may be valid yet such that the argument is not cogent for its audience.

\section{Notes}

1 Unless otherwise indicated, page references are to Trudy Govier, Problems in Argument Analysis and Evaluation, Foris Publications, Dordrecht-Holland/Providence RI - U.S.A., 1987.

2 Trudy Govier, A Practical Study of Argument, Wadsworth Publishing Company, Belmont, California, 1988. Hereafter cited as PSA.
3 PSA, Ch. 4. See esp. pp. 62-4.

4 The reference is to Ch. 4 of the 1985 edition of PSA. (See pp. 91-5). The 1988 edition also treats question-begging as a premise flaw: see pp. 90-2.

5 In one sense, then, an analogy for Govier is an analogical argument. But she also uses the word to mean a comparison between two cases. 
6 "Hence", because adequacy entails relevance: PSA 62.

7 "The thrust that underlies logical analogies is that of consistency... the consistency of treating relevantly similar cases similarly" (58).

8 Obviously she would say that a conductive argument can have an unstated conclusion. But would she say that a conductive argument can have a missing premise? Let the argument be of the form $\mathbf{P}$, so $\mathrm{C}$. And suppose that: (1) P is positively relevant to $C$; (2) Q is a negatively relevant counterconsideration which outweighs P; (3) the arguer believes, and is epistemically justified in believing, that not $Q$; (4) there is no negatively relevant counterconsideration besides Q. Given (2), the argument has an unsound inference. But given (1), (3) and (4), it is surely not a fallacy. And given (1), (2) and (4), it would have a sound inference if 'not Q' were added to it as a further premise. Ex hypothesi, it is false that not Q. But suppose that there is contextual evidence that the arguer believes that it is true that not $Q$. Under these conditions I believe Govier would sanction adding 'not Q' to the argument as a missing premise (though it would not improve the argument). Thus I believe she would say that a conductive argument can have a missing premise. At any rate, her views on missing premises and on conductive arguments appear to allow her to say this.

9 See note 6.

10 Premise (1) is not, pace Govier/Wellman, a claim that "is unverifiable independently of a judgment about the case at issue", because it is not a claim about that case in particular. It is a generalization. (The relevant claim about the case at issue is that things are (or are not) equal in that case.)

11 Paul K. Moser, Empirical Justification, D. Reidel Publishing Company, Dordrecht, Holland, 1985, p. 29.

12 Ibid., p. 44.

${ }^{13}$ I take this point from ibid., pp. 6-8.

14 Ibid., p. 8.

\section{DEREK ALLEN}

DEPARTMENT OF PHILOSOPHY

UNIVERSITY OF TORONTO TORONTO, ONTARIO MSS 1 H8 